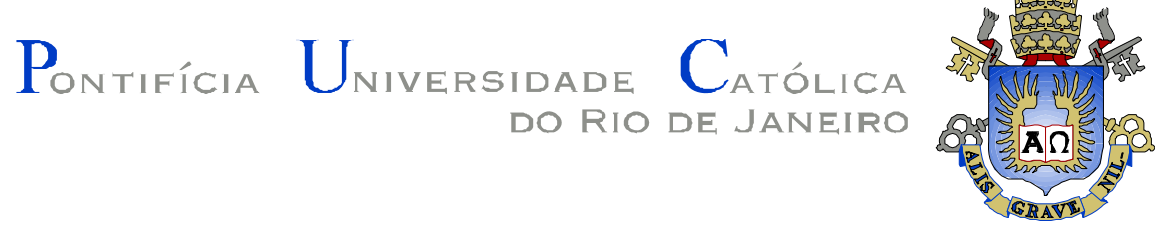

Cássia Maria Chaffin Guedes Pereira

\title{
A perdição criadora
}

Tese de Doutorado

Tese apresentada com requisito parcial para obtenção do grau de Doutor pelo Programa de Pós-Graduação em Psicologia Clínica do Departamento de Psicologia do Centro de Teologia e Ciências Humanas da PUC-Rio.

Orientador:

Profa. Monah Winograd

Coorientador:

Profa. Cláudia Maria Castro (in memoriam)

Rio de Janeiro

Fevereiro de 2011 


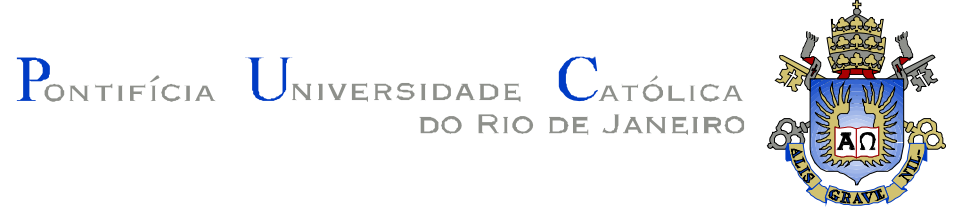

Cássia Maria Chaffin Guedes Pereira

\section{A perdição criadora}

Tese apresentada como requisito parcial para obtenção do grau de Doutor pelo Programa de Pós-Graduação em Psicologia Clínica do Departamento de Psicologia do Centro de Teologia e Ciências Humanas da PUC-Rio. Aprovada pela Comissão Julgadora abaixo assinada.

Profa. Monah Winograd

Orientadora Departamento de Psicologia - PUC-Rio

Profa. Flávia Sollero de Campos Departamento de Psicologia - PUC-Rio

Prof. José Thomaz Almeida Brum Duarte CCE - PUC-Rio

Prof. Aristides Ledesma Alonso Departamento de Línguas e Literatura - UERJ

Prof. Luiz Alberto Rezende de Oliveira CBPF/MCT

Profa. Denise Berruezo Portinari Coordenador Setorial de Pós-Graduação e Pesquisa do Centro de Teologia e Ciências Humanas - PUC-Rio 
Todos os direitos reservados. É proibida a reprodução total ou parcial do trabalho sem autorização do autor, do orientador e da universidade.

\section{Cássia Maria Chaffin Guedes Pereira} Graduou-se em Comunicação Social pela PUC-Rio em 1990. Obteve o título de Mestre em Comunicação Social pela Universidade Metodista de São Paulo em 1995. Graduou-se em Psicologia Clínica pela PUC-Rio em 2006. Psicanalista com formação pelo Centro de Arte e Psicanálise. Professora do departamento de Comunicação Social da PUC-Rio e das Faculdades Hélio Alonso (FACHA).

Ficha Catalográfica

Pereira, Cássia Maria Chaffin Guedes

A perdição criadora / Cássia Maria Chaffin Guedes Pereira ; orientadora: Monah Winograd ; coorientadora: Cláudia Maria Castro (in memoriam). 2011.

212 f. ; $30 \mathrm{~cm}$

Tese (doutorado)-Pontifícia Universidade Católica do Rio de Janeiro, Departamento de Psicologia, 2011.

Inclui bibliografia

1. Psicologia - Teses. 2. Pulsão de morte. 3. Perda. 4. Desordem. 5. Trauma. 6. Criação. 7. Perdição. 8. Desejo. 9. Trágico. I. Winograd, Monah. II. Castro, Claudia Maria Castro (in memoriam). III. Pontifícia Universidade Católica do Rio de Janeiro. Departamento de Psicologia. IV. Título. 
Ao meu mestre e analista,

Evandro Meirelles Santos,

pelo rigor de sua análise interminável, que franqueou a passagem para minha perdição criadora. 


\section{Agradecimentos}

A Monah Winograd e Cláudia Castro (in memoriam), pela acolhida e orientação precisa do trabalho;

A Evandro Meirelles Santos, pelo modo singular de ensino da psicanálise, que mantém obrigatória a relação entre teoria e vida;

A PUC-Rio, pela bolsa para a realização do doutorado, sem a qual este trabalho não poderia ter sido realizado;

A Flávia Sollero, pelo apoio imprescindível para a realização do doutorado;

A Lenivaldo Gomes, pela disponibilidade de ler e comentar o trabalho em diversos momentos de sua elaboração;

A José Thomaz Brum, pela leitura generosa da primeira versão da tese;

A Rosana Suarez, pela participação na banca de qualificação e pelos comentários específicos sobre Nietzsche;

A Luiz Alberto de Oliveira, pelas observações sobre a relação entre física e psicanálise proposta neste trabalho;

Aos colegas do Centro de Arte e Psicanálise (Cenapsi) - Cristiane Monteiro, Joana Ferry, Juliana Sobral, Glória Malvestitti, Raquel Braz, pelo estímulo à caminhada, e, em especial, a José Roberto Britto, pelas sempre inspiradoras lembranças de poemas e citações bíblicas;

A Rosa Alba de Oliveira, por aceitar o convite para compor a equipe de avaliação a tese;

A Marise Lira, do departamento de Comunicação Social da PUC-Rio, pela importante ajuda para a finalização do doutorado;

A Marcelina Andrade, pela boa vontade e bom humor presentes no trato com os alunos da pós-graduação do departamento de Psicologia da PUC-Rio; e a Sandra Lara, da DAR, pelo atendimento sempre gentil.

A Maria Cristina Ribas e Ana Lucia Enne, pelas estimulantes conversas em momentos importantes do curso;

Aos meus alunos da PUC, da Facha, e do grupo de estudos, por emprestarem seus ouvidos às minhas besteiras. 


\section{Resumo}

Pereira, Cássia Maria Chaffin Guedes; Winograd, Monah (orientador); Castro, Cláudia Maria (coorientador). A perdição criadora. Rio de Janeiro, 2011. 212p. Tese de Doutorado - Departamento de Psicologia, Pontifícia Universidade Católica do Rio de Janeiro.

Este trabalho apresenta a hipótese de que o processo analítico implica na perdição criadora. Com esta expressão busca destacar tanto a experiência da perda como o despertar do desejo, presentes na análise. Ambos provocam sensação de desordem, vivida como experiência traumática. No entanto, constituem condição necessária para a invenção de nova maneira de viver e perceber o mundo. Parte do conceito de pulsão de morte, formulado por Sigmund Freud e reelaborado pelos psicanalistas Jacques Lacan e MD Magno, para pensar o fenômeno da criação, tanto na cultura como na clínica psicanalítica. Ao associar a pulsão de morte ao caráter trágico da existência, o trabalho estabelece diálogo entre a psicanálise e o pensamento de Friedrich Nietzsche.

\section{Palavras-Chave} trágico.

Pulsão de morte; perda; desordem; trauma; criação; perdição; desejo; 


\section{Abstract}

Pereira, Cássia Maria Chaffin; Winograd, Monah (advisor); Castro, Cláudia Maria (co-advisor). The creative perdition. Rio de Janeiro, 2011. 212p. Doctorate Thesis - Departamento de Psicologia, Pontifícia Universidade Católica do Rio de Janeiro.

This thesis presents the hypothesis that psychoanalytic process implicates in creative perdition. By this expression it intends to emphasize the loss experience as much as the awakening of desire. Both incite a sensation of disorder, associated to a traumatic experience. Nevertheless, they are necessary to promote the invention of an original way of living. The thesis starts with the study of the death drive, formulated by Freud and elaborated anew by the psychoanalysts Jacques Lacan and MD Magno, in order to investigate the creation phenomenon, as related both to culture and psychoanalytic clinic. By associating death drive to the tragic character of existence, this thesis establishes a dialogue between psychoanalysis and Friedrich Nietzsche's thought.

\section{Keywords}

Death drive; loss; disorder; trauma; creation; perdition; desire; tragic. 


\section{Sumário}

1. Introdução: Perdição e cultura

2. Freud: pulsão e criação

2.1. Pulsão de morte e paz

2.1.1. A montagem da pulsão

2.2. Caos e criação

41

2.3. A economia da tentação 56

2.4. As travas à perdição 70

2.5. Os sistemas mentais $\quad 86$

2.6. Extravios da moral 100

3. Nietzsche: vontade de poder e criação 111

3.1. Experimento e tentação: o estilo nietzscheano 118

3.2. Trágico, sacrilégio e perdição 126

3.3. Amor fati: a saúde da doença 135

3.4. Genealogia e transmutação de valores 144

4. A clínica da perdição criadora 155

4.1. O trabalho da perda 162

4.1.1. Perda, trauma e morte 170

4.2. Do amor ao agalma 178

5. Conclusão: Repetição e criação 198

6. Referências Bibliográficas 203 
Eu estou perdido para o mundo Com o qual eu desperdicei muito tempo, E há tanto que ele nada recebe de mim Que pode bem pensar que estou morto!

Mas não me importo nada Se ele me crê morto,

Eu também nada posso dizer contra, Porque na realidade estou morto para o mundo.

Eu estou morto para a sua agitação, E descanso numa tranquila região!

Eu vivo, só, no meu paraíso, No meu amor, na minha canção!

Friedrich Rückert $^{1}$

\footnotetext{
${ }^{1}$ O poema - Estou perdido para o mundo (Ich Bin der Welt Abhanden Gekommen) - de Friedrich Rückert (1788-1866), inspirou Gustav Mahler (1860-1911) a compor uma de suas mais belas canções.
} 